\section{Fatores associados a acidentes de trabalho envolvendo trabalhadores informais do comércio}

\author{
Factors associated with work-related accidents in
} the informal commercial sector

\author{
Factores asociados con accidentes de trabajo \\ relacionados con los trabajadores informales \\ del sector comercial
}

\begin{abstract}
This study aimed to estimate the incidence of non-fatal work-related accidents in the informal commercial sector and analyze associated socio-demographic, occupational, workplace, and health factors, in a cross-sectional survey of 434 workers in the business district of Jequié, Bahia State, Brazil, in 2013. Logistic regression was used to assess factors associated with accidents. Incidence of accidents in the previous 12 months was estimated at $32.3 \%$, and multivariate analysis showed higher odds of accidents in male sex workers $(O R=1.61)$, young individuals $(O R=$ $4.62)$, meat or poultry workers ( $O R=9.55)$, and workers performing heavy physical work $(O R=$ 1.71). The results show the need for public policies to prevent accidents in the informal commercial sector
\end{abstract}

Occupational Accidents; Occupational Health; Commerce
Marcela Andrade Rios 1

Adriana Alves Nery 2

Polianna Alves Andrade Rios 2

Cezar Augusto Casotti 2

Jefferson Paixão Cardoso 2

\section{Resumo}

O estudo objetivou estimar a incidência de acidentes de trabalho não fatais sobre trabalhadores informais do comércio e analisar os fatores associados a esses eventos quanto aos aspectos sociodemográficos, ocupacionais, condições laborais e de saúde. Realizou-se estudo transversal com 434 trabalhadores em área comercial do Município de Jequié, Bahia, Brasil, em 2013. A regressão logística foi utilizada para avaliar os possíveis fatores associados ao acidente. Estimou-se a incidência de acidentes de trabalho (nos últimos 12 meses) em 32,3\%, e a análise multivariada revelou maior chance de ocorrência de acidente entre trabalhadores do sexo masculino $(O R=1,61)$, jovens $(O R=4,62)$, comerciantes de carnes/frangos $(O R=9,55)$ e aqueles que apresentam alto esforço físico $(O R=1,71)$. Com a análise dos resultados, verifica-se a necessidade de políticas públicas voltadas para a prevenção de acidentes no setor informal comércio.

Acidentes de Trabalho; Saúde do Trabalhador; Comércio 


\section{Introdução}

Os acidentes laborais constituem importantes agravos que atingem a saúde dos trabalhadores. Apresentam-se como um sério problema de saúde pública, já que o trabalho representa um papel significativo nas condições de vida e saúde da população, no provimento do sustento dos núcleos familiares e no movimento da economia de um país 1 .

Conceitua-se acidente de trabalho como o evento ocorrido no exercício de atividade laborativa, independentemente da situação empregatícia e previdenciária do acidentado, que acarreta dano à saúde, potencial ou imediato, provocando lesão corporal ou perturbação funcional que causa, direta ou indiretamente, morte, ou perda ou redução da capacidade para o trabalho ${ }^{2}$. Na concepção de Iwamoto et al. ${ }^{3}$, a caracterização legal dos acidentes de trabalho pode ser classificada em duas categorias: aqueles que ocorrem no ambiente de trabalho e os que acontecem no trajeto.

Embora essa problemática da ocorrência de acidentes de trabalho seja cada vez mais frequente na realidade do país, as estatísticas oficiais sobre tais agravos são limitadas. Esse quadro decorre dos grandes níveis de subnotificação, o que dificulta estimar a real situação dos eventos e agravos incidentes na população trabalhadora brasileira 4 .

Na economia informal do país, a situação é bem mais grave. Muitas vezes, as pessoas trabalham por conta própria, sem registro em carteira de trabalho, raramente se notificando a ocorrência de acidentes de trabalho. Ademais, os sistemas de informação de saúde do trabalhador no Brasil são limitados a casos graves, aqueles que necessitam de hospitalização, os casos de óbito e aqueles que, independentemente da gravidade, ocorrem em trabalhadores vinculados à Previdência Social.

A informalidade da economia assume distintos significados, dependentes, sobretudo, do significado de formalidade existente em cada país e período. $\mathrm{O}$ uso da expressão trabalho informal tem suas origens nos estudos realizados pela Organização Internacional do Trabalho (OIT) no âmbito do Programa Mundial de Emprego de 19725 .

Na América Latina, difundiram-se pelo menos duas perspectivas ou abordagens sobre a informalidade. Uma está relacionada com a lógica da sobrevivência em um contexto econômico adverso, no qual um excedente estrutural de mão de obra pressiona o mercado de trabalho, tornando a oferta de bons empregos insuficiente. Outra, historicamente mais recente, leva em con- sideração as mudanças na divisão internacional do trabalho na era da globalização, a adaptação das empresas modernas diante de uma demanda instável e a adoção de medidas de descentralização produtiva associadas com a subcontratação de empresas e de mão de obra 5 . Acrescenta-se, ainda, outra forma de perceber e caracterizar o setor informal, que o entende como sinônimo de ilegalidade 6 .

Para a OIT, no âmbito da América Latina e Caribe, a economia informal é composta por atividades, geradoras de renda, que se desenvolvem fora do âmbito normativo oficial, em mercados desregulamentados e competitivos, em que é difícil distinguir a diferença entre capital e trabalho. Portanto, o trabalho informal pode ser caracterizado como aquele que abarca atividades produtivas executadas à margem da lei, ou seja, fora da legislação trabalhista vigente em determinado país 7 .

Apesar das diferenças semânticas e/ou taxonômicas, pode-se identificar convergência de sentido para a definição do trabalho informal, que seria a precariedade das unidades de trabalho e das condições e relações laborais ${ }^{6}$. De maneira geral, as condições de trabalho no setor informal são perigosas e insalubres, observando-se nelas a presença de múltiplos fatores de risco para a saúde e a ausência de dispositivos e mecanismos básicos de proteção. A esses fatores, soma-se a falta de proteção legal advinda da informalização do contrato trabalhista, o descumprimento de normas básicas de segurança, a ausência de fiscalização, além da falta de cobertura do seguro social e de acidentes do trabalho ${ }^{4}$.

Nesse contexto, torna-se relevante analisar as características sociodemográficas, bem como as condições de saúde e laborais dos trabalhadores inseridos no setor informal da economia e tangenciados nas estatísticas oficiais.

O setor informal abarca diversos tipos de atividades econômicas, a exemplo do comércio. Este é um subsetor marcado por heterogeneidade de atividades desenvolvidas e que compreende uma infinidade de tarefas de diversas naturezas, que incluem desde a operação de máquinas registradoras, reposição de estoques em gôndolas e prateleiras, até o desenvolvimento de múltiplas funções 8 .

Outra característica do comércio informal concerne aos ambientes de trabalho, muitas vezes insalubres, sem preocupações com aspectos de segurança, higiene e desprovidos de instalações mínimas de satisfação das necessidades mais elementares dos trabalhadores. Além dessa problemática, as condições laborais são precárias, manifestadas na violação dos direitos traba- 
lhistas, na insegurança do posto e do ambiente de trabalho, no aumento do ritmo da produção e das exigências. Tais condições interferem na saúde dos trabalhadores e também no modo de agir, pensar, sentir e fazer 9 .

Nesse sentido, apreender as condições de trabalho é um desafio dialético, materialmente necessário, para qualificar a questão dos eventos adversos à saúde do trabalhador, caracterizando-os como elementos constitutivos da lógica reprodutiva do sistema capitalista ${ }^{9}$. O conhecimento sobre o que ocorre e o que pode ocorrer em um sistema produtivo é de extrema importância para analisar os eventos, compreender os riscos; além de nortear a implementação de normas de segurança para a saúde do trabalhador e de projetos sobre desenvolvimento de máquinas, equipamentos e produtos, o conhecimento pauta, ainda, a organização dos sistemas de gestão das empresas, com o objetivo de garantir o desenvolvimento tecnológico, melhorando as condições de trabalho 10 .

Este estudo objetivou estimar a incidência de acidentes de trabalho não fatais sobre trabalhadores informais do comércio e analisar os fatores associados a esses eventos quanto aos aspectos sociodemográficos, ocupacionais, condições laborais e de saúde.

\section{Material e métodos}

Trata-se de estudo transversal acerca dos acidentes de trabalho entre trabalhadores informais de área comercial do Município de Jequié, Bahia, Brasil, no ano de 2013.

Conforme apontam pesquisadores 11 , embora os estudos transversais sejam escolhidos para investigar casos prevalentes, é possível estimar a taxa de incidência em alguns casos, como em pesquisas para coletar informações sobre acidentes de trabalho em um determinado período de tempo. Uma vez que os acidentes de trabalho são de natureza súbita, aguda, circunscritas no tempo e com duração curta, serão sempre casos incidentes.

Foram incluídos no estudo os trabalhadores que desenvolvem atividades como comerciantes (camelôs, feirantes ou ambulantes) no Centro de Abastecimento Vicente Grilo, com idade igual ou superior a 14 anos de idade, sem registro em carteira de trabalho referente a essa atividade. Foram excluídos aqueles que, no mesmo local, desempenham as atividades de comércio com registro em carteira de trabalho, trabalhadores ambulantes cujas atividades são em local não especificado pela administração do Centro de Abastecimento e os carregadores.
O Centro de Abastecimento Vicente Grilo agrega grande parte de trabalhadores informais de diferentes ramos econômicos do município. $\mathrm{O}$ espaço é dividido em pavilhões, quadras e áreas para shopping popular, para barracas e para vendedores ambulantes, totalizando 1.404 unidades comerciais, segundo relatório geral disponibilizado pela administração do Centro de Abastecimento em 2012.

Como não havia estimativa do número de trabalhadores nessa área comercial, os pesquisadores realizaram, no mês de outubro de 2012, levantamento do quantitativo de trabalhadores informais com idade igual ou superior a 14 anos, em todas as unidades comerciais existentes no local. Assim, estimou-se um total de 1.304 trabalhadores.

O cálculo da amostra foi realizado no software Epi Info, versão 6.04 (Centers for Disease Control and Prevention, Atlanta, Estados Unidos), com as seguintes informações: incidência de acidentes de trabalho de $6,5 \% 12$, erro de estimação de $2 \%$ e nível de $95 \%$ de confiança, o que resultou no tamanho da amostra de 388 indivíduos. Para compensar eventuais perdas e para permitir um melhor nível de estratificação das variáveis independentes, o tamanho amostral foi acrescido em $20 \%$, totalizando-se 485 pessoas.

Os trabalhadores foram selecionados por meio de amostragem aleatória sistemática, obedecendo ao intervalo de três trabalhadores ( $\mathrm{a}=$ 3 ), segundo listagem de todos os sujeitos obtida pelo levantamento anteriormente realizado. Aqueles indivíduos selecionados que não foram encontrados após três visitas em dias diferentes, incluindo ao menos um sábado, foram substituídos pelo próximo da lista em ordem crescente. Consideraram-se perdas aqueles que se recusaram a participar do estudo.

Para padronização da coleta de dados, os entrevistadores receberam treinamento. $\mathrm{O}$ estudopiloto foi realizado um mês antes do início da coleta de dados, com 25 trabalhadores da feira livre de um bairro do município. Para a coleta propriamente, foi utilizado um formulário composto por cinco blocos, a fim de se obterem as seguintes informações: (i) sociodemográficas; (ii) ocupacionais; (iii) de condições de trabalho; (iv) de saúde; e (v) de aspectos referentes a acidentes de trabalho.

As informações sociodemográficas estudadas foram sexo, idade, situação conjugal, escolaridade, raça/cor, número de filhos e naturalidade. Os aspectos ocupacionais referiram-se ao tipo de mercadoria comercializada, jornada de trabalho diária, renda mensal e tempo de ocupação. Quanto às condições de trabalho, foram investigados os aspectos psicossociais do trabalho e a 
percepção de riscos à saúde no processo laboral. A mensuração dos aspectos psicossociais do trabalho baseou-se no modelo demanda-controle, utilizando-se questões do instrumento Job Content Questionnaire (JCQ), traduzido e validado para grupos ocupacionais no Brasil 13.

Para a construção do modelo demanda-controle, seguiram-se os procedimentos recomendados pelo manual do JCQ ${ }^{14}$. Para dicotomizar as respostas em dois níveis (baixo e alto), considerou-se a mediana do próprio grupo (demanda psicológica e controle sobre o trabalho) como ponto de corte. Após essa etapa, foram construídas as categorias de análise do modelo, combinando alta e baixa demanda psicológica com alto e baixo controle, refletindo as seguintes situações de trabalho: baixa exigência (combinação de baixa demanda e alto controle), trabalho passivo (baixa demanda e baixo controle), trabalho ativo (alta demanda e alto controle) e alta exigência (alta demanda e baixo controle) 13,15. Além desses aspectos, analisou-se, ainda, o esforço físico despendido no trabalho, dicotomizado em alto e baixo de acordo com a mediana; a satisfação com o trabalho no comércio; a satisfação com a capacidade para trabalhar e a avaliação da qualidade de vida.

Em relação às condições de saúde, foram investigadas as doenças autorreferidas, a procura por serviços de saúde nos últimos 12 meses e a percepção da própria saúde. A fim de verificar a ocorrência de acidentes de trabalho, foi questionado se o trabalhador havia sofrido algum tipo de acidente no desenvolvimento de suas atividades laborais, no comércio ou no trajeto casa-trabalho ou trabalho-casa, nos últimos 12 meses. Para caracterizar tais agravos, considerou-se o caso mais recente relatado pelo trabalhador, buscando-se a minimização do viés de memória.

Os acidentes de trabalho foram considerados, no estudo, como qualquer dano infligido ao corpo por transferência de energia durante a atividade laboral, ou deslocamento até o local do trabalho que envolvesse uma curta duração entre exposição e efeitos identificáveis após a ocorrência do evento/circunstância 16. Como se previa que a percepção e o reconhecimento de acidentes ocupacionais por trabalhadores do setor informal estivessem prejudicados pela predominância de uma visão previdenciária, demarcada por propósitos administrativos e compensatórios empregados pelo sistema nacional de seguridade social, antes de perguntar ao trabalhador se já havia sofrido algum acidente laboral, foi-lhe explicado o conceito de acidente, decidindo-se por uma abordagem indireta.

A incidência de acidentes de trabalho foi calculada dividindo-se o número de casos identi- ficados de acidentes de trabalho não fatais, no período de referência, pelo total da população do estudo. Nota-se que, embora o estudo seja transversal, o fato de acidentes serem eventos circunscritos no tempo permite a estimativa de casos incidentes, com base em informações referidas no passado 11 .

A ocorrência de acidentes de trabalho foi considerada como a variável dependente do estudo. As independentes foram aquelas relacionadas aos aspectos sociodemográficos, ocupacionais, condições de trabalho e de saúde.

A análise dos dados foi feita em três etapas. Inicialmente, realizou-se análise estatística descritiva, para caracterizar a população do estudo e os acidentes de trabalho referidos. Na segunda etapa, procedeu-se à verificação de fatores associados ao desfecho, iniciando-se com análise bivariada entre a variável dependente (acidentes de trabalho) e as variáveis independentes, para estimação do risco relativo (RR) e seu respectivo intervalo de 95\% de confiança (IC95\%). Na terceira etapa, foi realizada análise multivariada por meio do modelo de regressão logística. $\mathrm{Na}$ modelagem, inicialmente foram estimados modelos brutos, considerando valor de $\mathrm{p}<0,20$ para significância estatística, ou seja, para selecionar as variáveis que seguiriam para o modelo multivariado. Neste, foi adotado valor de $\mathrm{p}<$ 0,05 como nível de significância estatística. A estimação do modelo final, portanto, ocorreu a partir da verificação de pressupostos estatísticos de significância, assim como da importância teórica de cada variável estudada. As variáveis foram selecionadas com o método backward na modelagem final, e não foram testadas possíveis interações. $\mathrm{O}$ ajuste do modelo foi avaliado pela razão de máxima verossimilhança.

Os dados foram analisados no programa estatístico Stata/SE 12.0 (Stata Corp., College Station, Estados Unidos).

O estudo foi aprovado pelo Comitê de Ética em Pesquisa da Universidade Estadual do Sudoeste da Bahia, campus de Jequié, sob CAAE no 04755112.3.0000.0055. Foram seguidos os preceitos éticos, de acordo com a Resolução no 466/2012 17 do Conselho Nacional de Saúde. Todos os participantes assinaram o termo de consentimento livre e esclarecido.

\section{Resultados}

Foram entrevistados 434 trabalhadores informais do comércio de Jequié. A perda de 11,5\% ocorreu por recusas dos informantes.

Dos entrevistados, 140 referiram ter sofrido algum tipo de acidente de trabalho nos últimos 
12 meses, estimando-se, desse modo, uma incidência de 32,3\% (IC95\%: 27,9\%-36,9\%). Dentre os trabalhadores acidentados, 19 (13,6\%) informaram a ocorrência de mais de um acidente no período estudado.

As características sociodemográficas, ocupacionais, de condições laborais e de saúde de toda a população do estudo e daqueles que sofreram algum acidente de trabalho mostraram-se semelhantes, havendo diferenças somente quanto ao número de doenças autorreferidas e ao controle sobre o trabalho.

Os trabalhadores, em sua maior parte, eram do sexo masculino, com idade entre 30 a 59 anos, solteiros, com Ensino Fundamental incompleto e autodeclarados pardos/pretos. Uma expressiva maioria dos trabalhadores desempenha atividades laborais somente no comércio informal, sendo este a principal fonte de renda da família (87,9\% a $90 \%$ ) (Tabela 1$)$.

Dentre os acidentes de trabalho relatados, aqueles classificados como típicos sobressaíram sobre os de trajeto $(82,1 \%)$. O principal tipo de acidente foi corte com faca $(41,4 \%)$, seguido por lesão causada por parte estrutural da barraca $(15,7 \%)$, quedas $(8,6 \%)$ e acidente com motocicleta $(7,1 \%)$. Os acidentes causaram lesão em $90,7 \%$ dos trabalhadores, especialmente nos membros superiores $(65,3 \%)$, sendo corte o principal tipo de lesão relatado $(65,3 \%)$, seguido por hematoma/edema $(18,9 \%)$.

Na análise bivariada (Tabela 2), foi encontrada associação entre acidentes de trabalho e sexo, idade, situação conjugal e escolaridade. A incidência de acidentes foi $32 \%$ maior no sexo masculino que no feminino. Entre os trabalhadores com idade inferior a 30 anos, o risco de sofrer acidentes foi 3,53 maior, quando comparados àqueles com idade igual ou superior a 60 anos $(p=0,000)$. Em relação aos aspectos referentes às condições de saúde, nenhuma variável desse grupo mostrou-se associada ao acidente de trabalho, conforme apresentado na Tabela 3.

Quanto ao bloco de aspectos ocupacionais e de condições de trabalho, as variáveis associadas na etapa bivariada foram: mercadoria comercializada, renda mensal, demanda psicológica, controle sobre o trabalho, esforço físico, modelo demanda-controle, satisfação com o trabalho, percepção dos fatores de risco e capacidade para o trabalho (Tabela 4 ).

Na variável tipo de mercadoria comercializada, a categoria de trabalhadores do comércio de carnes/frangos apresentou incidência de acidente $46,7 \%$ maior do que os comerciantes de alimentos e bebidas ( $R R=2,88$ : IC95\%: $1,67 \%-4,99 \%)$.
Na modelagem, as variáveis que se mostraram associadas a acidente de trabalho e que seguiram para o modelo multivariado foram: sexo, idade, tipo de mercadoria comercializada, modelo de demanda-controle e satisfação com o trabalho.

O modelo logístico final, apresentado na Tabela 5, incluiu variáveis que expressam características sociodemográficas, ocupacionais e de condições de trabalho. Foi observada maior chance de ocorrência de acidente de trabalho entre pessoas com as seguintes características: sexo masculino (OR $=1,61$; IC95\%: $1,00 \%$ $2,58 \%$ ), jovem (OR = 4,62; IC95\%: 1,82\%-11,73\%), que comercializam mercadorias do tipo carnes/ frangos (OR = 9,55; IC95\%: 4,12\%-22,16\%) e que despendem alto esforço físico $(\mathrm{OR}=1,71$; IC95\%: $1,04 \%-2,81 \%$ ).

Embora a variável percepção de risco não tenha apresentado associação no modelo não ajustado, ela seguiu para o modelo final em virtude da sua importância teórica. Já a categoria alta exigência do modelo demanda-controle apresentou valor com significância estatística na modelagem bruta, porém o mesmo não foi encontrado na modelagem final.

Ressalta-se, ainda, que, por relevância teórica, algumas variáveis foram mantidas no modelo final, obedecendo-se à seguinte ordem de importância: demanda física, controle sobre o trabalho e demanda psicológica. Após as verificações dos melhores ajustes, as variáveis demanda física e demanda psicológica foram mantidas

As variáveis sexo, idade, mercadoria comercializada e esforço físico mostraram-se como fatores de risco para os acidentes de trabalho. Percepção de fatores de riscos à saúde presentes no processo de trabalho e demanda psicológica mostraram-se como fatores de proteção. Os indivíduos que relataram não perceber riscos à saúde no trabalho apresentaram chance $54 \%$ menor de sofrer acidentes de trabalho quando comparados àqueles que percebem algum risco. Para a demanda psicológica, os indivíduos classificados com alta demanda mostraram chance $44 \%$ menor de sofrer o desfecho que aqueles com baixa demanda psicológica.

\section{Discussão}

A incidência de acidentes de trabalho estimada no presente estudo é de $32,3 \%$, no entanto, pela existência de poucos estudos abordando os trabalhadores informais do comércio 18 , especialmente no que tange aos fatores associados aos acidentes de trabalho, existe certa dificuldade nas comparações diretas com outras pesquisas. 
Características da população do estudo, segundo variáveis sociodemográficas, ocupacionais e relacionadas às condições laborais e de saúde. Jequié, Bahia, Brasil, 2013.

\begin{tabular}{|c|c|c|c|c|}
\hline \multirow[t]{2}{*}{ Variáveis } & \multicolumn{2}{|c|}{ População total do estudo } & \multicolumn{2}{|c|}{ Acidentados } \\
\hline & $\mathrm{n}$ & $\%$ & $\mathrm{n}$ & $\%$ \\
\hline \multicolumn{5}{|l|}{ Sexo } \\
\hline Feminino & 197 & 45,6 & 54 & 38,6 \\
\hline Masculino & 237 & 54,6 & 86 & 61,4 \\
\hline \multicolumn{5}{|l|}{ Idade (anos) } \\
\hline$<30$ & 60 & 13,8 & 7 & 5,0 \\
\hline $30-59$ & 265 & 61,1 & 88 & 62,9 \\
\hline 60 e mais & 109 & 25,1 & 45 & 32,1 \\
\hline \multicolumn{5}{|l|}{ Situação conjugal } \\
\hline Solteiro & 175 & 40,4 & 66 & 47,1 \\
\hline Casado/União estável & 221 & 51,0 & 62 & 44,3 \\
\hline Divorciado/Separado/Viúvo & 37 & 8,5 & 12 & 8,6 \\
\hline \multicolumn{5}{|l|}{ Escolaridade } \\
\hline Ensino Fundamental & 285 & 65,7 & 80 & 57,1 \\
\hline A partir do Ensino Médio & 149 & 34,3 & 60 & 42,9 \\
\hline \multicolumn{5}{|l|}{ Raça/Cor } \\
\hline Branca & 75 & 17,3 & 20 & 14,3 \\
\hline Preta/Parda & 306 & 70,5 & 105 & 75,0 \\
\hline Outras & 53 & 12,2 & 15 & 10,7 \\
\hline \multicolumn{5}{|c|}{ Procurou por serviços de saúde nos últimos 12 meses } \\
\hline Sim & 277 & 63,8 & 87 & 62,1 \\
\hline Não & 157 & 36,2 & 53 & 37,9 \\
\hline \multicolumn{5}{|l|}{ Percepção da saúde } \\
\hline Boa & 278 & 64,1 & 85 & 60,7 \\
\hline Regular & 142 & 32,7 & 50 & 35,7 \\
\hline Ruim & 14 & 3,2 & 5 & 3,6 \\
\hline \multicolumn{5}{|c|}{ Número de morbidades autorreferidas } \\
\hline Nenhuma & 119 & 27,4 & 36 & 25,7 \\
\hline Somente uma & 102 & 23,5 & 37 & 26,4 \\
\hline Mais de uma & 213 & 49,1 & 67 & 47,9 \\
\hline \multicolumn{5}{|l|}{ Mercadoria comercializada } \\
\hline Alimentos/Bebidas & 99 & 22,8 & 24 & 17,7 \\
\hline Frutas e verduras & 171 & 39,4 & 42 & 30,0 \\
\hline Outros tipos & 110 & 25,3 & 35 & 25,0 \\
\hline Carnes e frangos & 54 & 12,4 & 39 & 27,9 \\
\hline \multicolumn{5}{|l|}{ Jornada diária de trabalho (horas) } \\
\hline Até 8 & 107 & 24,7 & 31 & 22,1 \\
\hline Mais de 8 & 327 & 75,3 & 109 & 77,9 \\
\hline \multicolumn{5}{|c|}{ Renda média mensal (salário mínimo) } \\
\hline Até 1 & 232 & 53,5 & 71 & 50,7 \\
\hline Superior a 1 & 202 & 46,5 & 69 & 49,3 \\
\hline \multicolumn{5}{|l|}{ Demanda psicológica } \\
\hline Baixa & 221 & 50,9 & 84 & 60,0 \\
\hline Alta & 213 & 49,1 & 56 & 40,0 \\
\hline \multicolumn{5}{|l|}{ Controle sobre o trabalho } \\
\hline Baixo & 236 & 54,4 & 61 & 43,6 \\
\hline Alto & 198 & 45,6 & 79 & 56,4 \\
\hline
\end{tabular}


Tabela 1 (continuação)

\begin{tabular}{|c|c|c|c|c|}
\hline \multirow[t]{2}{*}{ Variáveis } & \multicolumn{2}{|c|}{ População total do estudo } & \multicolumn{2}{|c|}{ Acidentados } \\
\hline & $\mathrm{n}$ & $\%$ & $\mathbf{n}$ & $\%$ \\
\hline \multicolumn{5}{|l|}{ Esforço físico } \\
\hline Baixo & 215 & 49,5 & 62 & 44,3 \\
\hline Alto & 219 & 50,5 & 78 & 55,7 \\
\hline \multicolumn{5}{|c|}{ Modelo de demanda controle } \\
\hline Baixa exigência & 107 & 24,7 & 27 & 19,3 \\
\hline Trabalho passivo & 114 & 26,3 & 34 & 24,3 \\
\hline Trabalho ativo & 91 & 21,0 & 29 & 20,7 \\
\hline Alta exigência & 122 & 28,1 & 50 & 35,7 \\
\hline \multicolumn{5}{|c|}{ Satisfação com o trabalho } \\
\hline Muito satisfeito & 79 & 18,2 & 20 & 14,3 \\
\hline Satisfeito & 305 & 70,3 & 99 & 70,7 \\
\hline Insatisfeito & 50 & 11,5 & 21 & 20,0 \\
\hline
\end{tabular}

Por esse motivo, recorreu-se a publicações mais recentes de estudos realizados com trabalhadores brasileiros de diversos setores econômicos.

Como a ocorrência de acidentes de trabalho foi referida pelos entrevistados, ao se requerer que relembrassem os últimos 12 meses, é possível que o número de acidentes esteja subestimado, havendo a possibilidade de que os acidentes menos graves não tenham sido relatados. Além disso, devido ao desenho do estudo, existe a possibilidade de viés de prevalência, permitindo somente avaliar os acidentes naqueles indivíduos que estavam presentes no local de trabalho nos meses em que foi realizada a coleta de dados da pesquisa.

Quanto às características dos agravos, a ocorrência de acidentes típicos foi superior à dos acidentes de trajeto. O tipo de acidente classificado como corte com faca foi o mais frequentemente relatado. Acredita-se que esta causa apresentouse expressiva porque as facas são ferramentas de trabalho geralmente utilizadas em feiras por diversos tipos de trabalhadores, em diferentes ocupações, sendo de fácil manejo e usadas em pequenos serviços que requerem pouca habilidade e experiência. Assim, o trabalhador, raramente, apresenta capacitação adequada para identificar se elas estão em boa condição de uso e quais os tipos de cuidados devem ser tomados ao manuseá-las 19.

Dentre os acidentes de trajeto, o mais frequente foi causado pelo uso da motocicleta, o que pode estar relacionado ao deslocamento para o trabalho ou de volta para a residência com acesso mais rápido ao destino.

No que concerne à análise dos fatores associados, a maior incidência de acidentes de tra- balho no sexo masculino e em adultos jovens também foi encontrada em outros estudos, com populações de diferentes ocupações formais ou informais 20,21,22,23. A maior incidência em trabalhadores menores de 30 anos pode ser atribuída à elevada participação dos jovens na força de trabalho desse setor, uma vez que o comércio possibilita a inserção do trabalhador em inúmeras funções não especializadas e de baixa remuneração, que, em geral, não requerem qualificação profissional. Isso faz com que o comércio seja um fator atrativo para os jovens inexperientes que almejam obter um posto de trabalho com mais facilidade 24 .

Não houve associação entre o acidente e a escolaridade. Como o nível de especialização das tarefas é pequeno, a escolaridade pode não ser uma condição para sua realização. De forma semelhante, esse fato também foi inferido por pesquisadores 25 ao estudar a prevalência de acidentes de trabalho em zona rural, onde muitos trabalhadores desenvolvem suas atividades em condições precárias quanto às leis trabalhistas. Também não foi encontrada associação com raça/cor.

Para o tipo de ocupação, encontrou-se forte associação estatística entre os acidentes e o trabalho como açougueiro. Uma das tarefas prestadas por trabalhadores que comercializam carnes e frangos envolve talhar e cortar as mercadorias com a utilização de ferramentas, motorizadas ou não, que podem levar ao risco de lesões nas mãos e nos dedos. Outro fator que deve ser abordado é a pressão da produtividade, visto que vendedores do comércio, em sua grande maioria, são os proprietários da unidade comercial e apresentam sua renda mensal de 
Análise descritiva bivariada de fatores sociodemográficos, condições de saúde e a ocorrência de acidentes de trabalho não fatais em trabalhadores informais do comércio. Jequié, Bahia, Brasil, 2013.

\begin{tabular}{|c|c|c|c|c|}
\hline Variáveis sociodemográficas e de condições de saúde & $\%$ & RR & IC95\% & Valor de $\mathrm{p}$ \\
\hline \multicolumn{5}{|l|}{ Sexo } \\
\hline Feminino & 27,40 & 1,00 & & \\
\hline Masculino & 36,30 & 1,32 & $0,99-1,75$ & 0,0489 \\
\hline \multicolumn{5}{|l|}{ Idade (anos) } \\
\hline$<30$ & 41,30 & 3,53 & $1,58-9,30$ & 0,000 \\
\hline $30-59$ & 33,20 & 2,85 & $1,32-7,28$ & 0,001 \\
\hline 60 e mais & 11,70 & 1,00 & & \\
\hline \multicolumn{5}{|l|}{ Situação conjugal } \\
\hline Solteiro & 37,71 & 1,33 & $0,91-1,98$ & 0,063 \\
\hline Casado & 28,24 & 1,00 & & \\
\hline Divorciado/Separado/Viúvo & 29,21 & 1,03 & $0,62-1,70$ & 0,440 \\
\hline \multicolumn{5}{|l|}{ Escolaridade } \\
\hline Ensino Fundamental & 28,42 & 0,72 & $0,55-0,94$ & 0,018 \\
\hline A partir do Ensino Médio & 39,60 & 1,00 & & \\
\hline \multicolumn{5}{|l|}{ Raça/Cor } \\
\hline Branca & 26,67 & 1,00 & & \\
\hline Preta/Parda & 34,31 & 1,28 & $0,79-2,19$ & 0,151 \\
\hline Outras & 28,30 & 1,06 & $0,50-2,18$ & 0,428 \\
\hline \multicolumn{5}{|l|}{ Avaliação da qualidade de vida } \\
\hline Boa & 32,88 & 1,00 & & \\
\hline Regular & 30,08 & 0,91 & $0,61-1,35$ & 0,327 \\
\hline Ruim & 36,54 & 1,12 & $0,43-2,39$ & 0,368 \\
\hline \multicolumn{5}{|l|}{ Procurou por serviços de saúde nos últimos 12 meses } \\
\hline Sim & 31,29 & 1,00 & & \\
\hline Não & 33,97 & 1,08 & $0,82-1,43$ & 0,566 \\
\hline \multicolumn{5}{|l|}{ Percepção de saúde } \\
\hline Boa & 30,58 & 1,00 & & \\
\hline Regular & 35,21 & 1,15 & $0,79-1,65$ & 0,213 \\
\hline Ruim & 35,71 & 1,16 & $0,36-2,83$ & 0,348 \\
\hline \multicolumn{5}{|l|}{ Número de morbidades autorreferidas } \\
\hline Nenhuma & 30,25 & 1,00 & & \\
\hline Somente uma & 36,27 & 1,19 & $0,74-1,95$ & 0,220 \\
\hline Mais de uma & 31,46 & 1,03 & $0,68-1,60$ & 0,429 \\
\hline
\end{tabular}

IC95\%: intervalo de 95\% de confiança; RR: risco relativo.

acordo com a quantidade de mercadoria vendida, ou seja, depende do valor da venda/faturamento. Essa variação de renda, a depender da produtividade, pode proporcionar esgotamento tanto físico quanto psicológico, aumentando suas chances de se acidentar 26 .

Quanto aos aspectos relativos às condições de saúde, estas não evidenciaram associações estatisticamente significantes com os acidentes de trabalho. Já no que se refere às condições de trabalho, encontrou-se associação estatisticamente significante, na etapa bivariada da aná- lise de regressão, entre acidente de trabalho e o modelo demanda-controle, na categoria alta exigência no trabalho. Entretanto, a variável modelo demanda-controle não permaneceu na modelagem final.

Os trabalhadores classificados como alta exigência possuem o risco potencial de distúrbios de ordem psicológica e de doença física à medida que o trabalho se desenvolve. As características de alta demanda combinada com baixo controle são intensificadas, havendo, ainda, possibilidade de repercussão sobre a saúde, como desgaste 
Tabela 3

Análise descritiva bivariada de variáveis ocupacionais e a ocorrência de acidentes de trabalho não fatais em trabalhadores informais do comércio. Jequié, Bahia, Brasil, 2013.

\begin{tabular}{|c|c|c|c|c|}
\hline Variáveis ocupacionais & $\%$ & RR & IC95\% & Valor de $\mathrm{p}$ \\
\hline \multicolumn{5}{|c|}{ Mercadoria comercializada } \\
\hline Alimentos/Bebidas & 25,00 & 1,00 & & \\
\hline Frutas e verduras & 24,14 & 0,96 & $0,57-1,67$ & 0,441 \\
\hline Outros tipos & 32,43 & 1,29 & $0,75-2,27$ & 0,163 \\
\hline Carnes e frangos & 71,70 & 2,88 & $1,67-4,99$ & 0,000 \\
\hline \multicolumn{5}{|c|}{ Jornada diária de trabalho (horas) } \\
\hline Até 8 & 28,97 & 1,00 & & \\
\hline Mais de 8 & 33,33 & 1,15 & $0,83-1,60$ & 0,402 \\
\hline \multicolumn{5}{|c|}{ Renda média mensal (salário mínimo) } \\
\hline Superior a 1 & 30,60 & 1,00 & & \\
\hline Até 1 & 37,12 & 1,21 & $0,82-1,77$ & 0,150 \\
\hline Não informado & 28,57 & 0,93 & $0,53-1,55$ & 0,401 \\
\hline \multicolumn{5}{|l|}{ Possui outro emprego } \\
\hline Não & 33,25 & 1,00 & & \\
\hline $\operatorname{Sim}$ & 25,45 & 0,76 & $0,48-1,23$ & 0,248 \\
\hline \multicolumn{5}{|c|}{ Tempo de ocupação no comércio informal (anos) } \\
\hline$<4$ & 25,00 & 1,00 & & \\
\hline $4-15$ & 33,96 & 1,36 & $0,66-3,24$ & 0,208 \\
\hline$>15$ & 30,66 & 1,23 & $0,56-3,02$ & 0,310 \\
\hline \multicolumn{5}{|l|}{ Demanda psicológica } \\
\hline Baixa & 35,59 & 1,00 & & \\
\hline Alta & 28,28 & 0,79 & $0,60-1,05$ & 0,104 \\
\hline \multicolumn{5}{|l|}{ Controle sobre o trabalho } \\
\hline Baixo & 27,60 & 1,00 & & \\
\hline Alto & 37,09 & 1,34 & $1,02-1,77$ & 0,034 \\
\hline \multicolumn{5}{|l|}{ Esforço físico } \\
\hline Baixo & 28,84 & 1,00 & & \\
\hline Alto & 35,62 & 1,23 & $0,94-1,63$ & 0,130 \\
\hline \multicolumn{5}{|c|}{ Modelo demanda-controle } \\
\hline Baixa exigência & 25,23 & 1,00 & & \\
\hline Trabalho passivo & 29,82 & 1,18 & $0,69-2,03$ & 0,260 \\
\hline Trabalho ativo & 31,87 & 1,26 & $0,72-2,21$ & 0,192 \\
\hline Alta exigência & 40,98 & 1,62 & $0,99-2,69$ & 0,020 \\
\hline \multicolumn{5}{|l|}{ Satisfação com o trabalho } \\
\hline Muito satisfeito & 25,32 & 1,00 & & \\
\hline Satisfeito & 32,46 & 1,28 & $0,79-2,19$ & 0,156 \\
\hline Insatisfeito & 42,00 & 1,66 & $0,85-3,22$ & 0,054 \\
\hline \multicolumn{5}{|c|}{ Percepção de fatores de risco } \\
\hline $\operatorname{Sim}$ & 35,37 & 1,00 & & \\
\hline Não & 22,64 & 0,64 & $0,44-0,94$ & 0,014 \\
\hline \multicolumn{5}{|c|}{ Satisfação com a capacidade para o trabalho } \\
\hline Satisfeito & 31,32 & 1,00 & & \\
\hline Insatisfeito & 42,86 & 1,37 & $0,61-2,69$ & 0,182 \\
\hline Regular & 34,69 & 1,10 & $0,62-1,85$ & 0,337 \\
\hline
\end{tabular}

IC95\%: intervalo de 95\% de confiança; RR: risco relativo. 
Análise de regressão logística com odds ratio (OR) não ajustadas, intervalos de 95\% de confiança (IC95\%) e valor de p da associação entre acidentes de trabalho e variáveis sociodemográficas e ocupacionais. Jequié, Bahia, Brasil, 2013.

\begin{tabular}{|c|c|c|c|}
\hline Variáveis & OR bruto & IC95\% & Valor de $p$ \\
\hline \multicolumn{4}{|l|}{ Variáveis sóciodemográficas } \\
\hline \multicolumn{4}{|l|}{ Sexo } \\
\hline Feminino & 1,00 & & \\
\hline Masculino & 1,50 & $1,00-2,27$ & 0,049 \\
\hline \multicolumn{4}{|l|}{ Idade (anos) } \\
\hline$<30$ & 5,32 & $2,21-12,77$ & 0,002 \\
\hline $30-59$ & 3,76 & $1,64-8,62$ & 0,000 \\
\hline 60 e mais & 1,00 & & \\
\hline \multicolumn{4}{|l|}{ Situação conjugal } \\
\hline Solteiro & 1,53 & $0,97-2,42$ & 0,062 \\
\hline Casado & 1,00 & & \\
\hline Divorciado/Separado/Viúvo & 1,04 & $0,59-1,84$ & 0,869 \\
\hline \multicolumn{4}{|l|}{ Escolaridade } \\
\hline Ensino Fundamental & 0,60 & $0,39-0,91$ & 0,018 \\
\hline A partir do Ensino Médio & 1,00 & & \\
\hline \multicolumn{4}{|l|}{ Variáveis ocupacionais } \\
\hline \multicolumn{4}{|l|}{ Mercadoria comercializada } \\
\hline Alimentos/Bebidas & 1,00 & & \\
\hline Frutas e verduras & 0,95 & $0,53-1,70$ & 0,875 \\
\hline Outros tipos & 1,44 & $0,78-2,64$ & 0,241 \\
\hline Carnes e frangos & 7,6 & $3,57-16,17$ & 0,000 \\
\hline \multicolumn{4}{|c|}{ Renda média mensal (salário mínimo) } \\
\hline Superior a 1 & 1,00 & $0,50-1,63$ & 0,745 \\
\hline Até 1 & 1,33 & $0,85-2,09$ & 0,204 \\
\hline \multicolumn{4}{|l|}{ Não informado } \\
\hline \multicolumn{4}{|l|}{ Demanda psicológica } \\
\hline Baixa & 1,00 & & \\
\hline Alta & 0,71 & $0,47-1,07$ & 0,105 \\
\hline \multicolumn{4}{|l|}{ Controle sobre o trabalho } \\
\hline Baixo & 1,00 & & \\
\hline Alto & 1,54 & $1,03-2,31$ & 0,319 \\
\hline \multicolumn{4}{|l|}{ Modelo demanda controle } \\
\hline Baixa exigência & 1,00 & & \\
\hline Trabalho passivo & 1,25 & $0,69-2,27$ & 0,446 \\
\hline Trabalho ativo & 1,38 & $0,74-2,57$ & 0,302 \\
\hline Alta exigência & 2,05 & $1,16-3,62$ & 0,012 \\
\hline \multicolumn{4}{|l|}{ Satisfação com o trabalho } \\
\hline Muito satisfeito & 1,00 & & \\
\hline Satisfeito & 1,41 & $0,80-2,48$ & 0,223 \\
\hline Insatisfeito & 2,13 & $1,00-4,55$ & 0,049 \\
\hline \multicolumn{4}{|l|}{ Percepção de fatores de risco } \\
\hline Sim & 1,00 & & \\
\hline Não & 0,53 & $0,911-2,04$ & 0,131 \\
\hline \multicolumn{4}{|c|}{ Satisfação com a capacidade para o trabalho } \\
\hline Satisfeito & 1,00 & & \\
\hline Insatisfeito & 1,64 & $0,67-4,01$ & 0274 \\
\hline Regular & 1,16 & $0,62-2,18$ & 0,634 \\
\hline
\end{tabular}


Modelo final de regressão logística multivariada da associação entre ocorrência de acidentes de trabalho e as variáveis independentes do estudo. Jequié, Bahia, Brasil, 2013.

\begin{tabular}{|c|c|c|c|}
\hline Variáveis & OR ajustado & IC95\% & Valor de $\mathrm{p}$ \\
\hline \multicolumn{4}{|l|}{ Sexo } \\
\hline Masculino & 1,61 & $1,00-2,58$ & 0,046 \\
\hline \multicolumn{4}{|l|}{ Idade (anos) } \\
\hline$<30$ & 4,62 & $1,82-11,73$ & 0,001 \\
\hline $30-59$ & 2,73 & $1,13-6,56$ & 0,025 \\
\hline \multicolumn{4}{|c|}{ Mercadoria comercializada } \\
\hline Frutas e verduras & 0,90 & $0,48-1,68$ & 0,755 \\
\hline Outros tipos & 1,85 & $0,93-3,65$ & 0,076 \\
\hline Carnes e frangos & 9,55 & $4,12-22,16$ & 0,000 \\
\hline \multicolumn{4}{|c|}{ Percepção de fatores de risco } \\
\hline Não & 0,46 & $0,26-0,84$ & 0,012 \\
\hline \multicolumn{4}{|l|}{ Esforço físico } \\
\hline Alto & 1,71 & $1,04-2,81$ & 0,032 \\
\hline \multicolumn{4}{|l|}{ Demanda psicológica } \\
\hline Alta & 0,66 & $0,41-1,07$ & 0,097 \\
\hline
\end{tabular}

IC95\%: intervalo de 95\% de confiança; OR: odds ratio.

Categorias de referência para as variáveis: $\operatorname{sexo}=$ feminino; idade $=60$ anos e mais; mercadoria comercializada $=$ alimentos e bebidas; percepção de fatores de risco = sim; esforço físico = alto; demanda psicológica = baixa .

psicológico, fadiga, ansiedade e depressão ${ }^{13}$. A demanda psicológica caracteriza-se pelas exigências que o trabalhador enfrenta no seu trabalho quanto a concentração, ritmo, tempo para realização das tarefas, entre outras. O controle considera a possibilidade de o trabalhador tomar decisões acerca do seu trabalho e também o nível de habilidade ou criatividade requerida para a tarefa; considera, ainda, a flexibilidade que permite ao trabalhador decidir quais habilidades empregar 13,15.

Na literatura, é possível encontrar estudos abordando a associação de trabalho de alta exigência com a prevalência de doenças relacionadas ao trabalho 15,27,28,29. Todavia, na última década, nas bases de dados LILACS e MEDLINE, não foram encontradas publicações que relacionassem acidentes laborais com a alta exigência no trabalho, verificada por meio do modelo demanda-controle.

A principal hipótese testada pela utilização das questões do JCQ é que reações adversas à saúde acontecem devido ao desgaste psicológico que afeta os trabalhadores. A exposição simultânea a elevadas demandas psicológicas e escassa amplitude de decisão sobre o seu processo de trabalho (controle) caracteriza o trabalho como sendo de alta exigência 13 .

Uma segunda hipótese do modelo decorre do que viria a ser um "efeito positivo" do estres- se. Poderia advir um comportamento ativo, com motivação, novas aprendizagens e um padrão de enfrentamento positivo, sob condições simultaneamente de demandas psicológicas e amplitude de decisão elevadas (trabalhos ativos) ${ }^{13}$.

A demanda psicológica, especificamente, refere-se às exigências psicológicas que o trabalhador enfrenta na realização das suas tarefas: concentração intensa, pressão do tempo para realização de tarefas, ritmo e volume de tarefas a serem realizadas 13,30. No presente estudo, com a análise da demanda psicológica na modelagem final, verificou-se que esta variável, associada aos acidentes de trabalho, comporta-se como um fator de proteção. Isso significa que os trabalhadores que desenvolvem suas atividades com maior nível de atenção, concentração e pressão de tempo tiveram menor chance de se acidentar no trabalho.

No que se refere aos aspectos psicossociais do trabalho, encontrou-se, na presente pesquisa, associação estatisticamente significante entre acidentes de trabalho e esforço físico. Estudos mostram que as condições laborais, incluindo elevado esforço físico exigido na execução de atividades laborais, levam a uma diminuição da capacidade de trabalho, consequentemente expondo o trabalhador a maiores riscos de acidentes 31,32. No comércio, observam-se condições insatisfatórias quanto aos aspectos ergonômicos e 
esforços físicos, tais como posturas inadequadas, transporte manual de objetos, uso de força, realização de movimentos repetitivos, deambulação frequente e trabalho em pé, condições que podem levar os indivíduos a altas exigência físicas.

Variável também estatisticamente associada aos acidentes de trabalho, a não percepção dos fatores de risco à saúde no processo de trabalho apresenta-se como um fator de proteção para os acidentes de trabalho. Tal achado deve ser visualizado com cautela, uma vez que a literatura aponta a percepção do risco como fator importante para a adoção de comportamentos preventivos 33,34 .

É importante considerar que as percepções de riscos dos trabalhadores podem sofrer processos de enviesamento. São tendencialmente construídas com base nas experiências vividas nos locais de trabalho, estruturando o modelo de representações dos atores sociais. Fundam-se no desenrolar das práticas quotidianas do mundo laboral, sendo mais ou menos influenciadas pelos discursos e pelas práticas produzidas no meio, bem como pelos fatores político-ideológicos dos sujeitos que visualizam, percebem e recebem a informação sobre os riscos organizacionais 35 .

Outro fator importante que parece influenciar as percepções de riscos no trabalho é o contato regular dos trabalhadores com as próprias situações de risco, isto é, alguns autores defendem que a exposição continuada a determinados tipos de risco tende a ter como efeito a diminuição ou a subavaliação da sua percepção 36 .

Desse modo, o trabalhador informal do comércio que já sofreu algum acidente de trabalho pode perceber mais claramente os riscos, pois estes o levaram ao evento, do que aquele que nunca sofreu qualquer tipo de acidente. Além de outros aspectos, a percepção de riscos no trabalho está associada a dimensões como a maior ou menor proximidade dos acidentes no tempo e a distância no espaço geográfico do sinistro ${ }^{35}$. Portanto, a aprendizagem com os acidentes pode também influenciar as percepções de riscos 37 .

Quanto às condições de saúde, não foi encontrada associação estatisticamente significante com os acidentes de trabalho, o que pode demonstrar que a existência de morbidades, melhor percepção da saúde e procura pelos serviços de saúde não interferem na ocorrência de acidentes laborais no comércio informal.

Com base nos resultados encontrados, ressalta-se a importância do conhecimento real dos agravos que atingem os trabalhadores informais, uma vez que notificações e investigações dos acidentes de trabalho estão entre os principais requisitos para as ações de vigilância em saúde do trabalhador.

É importante destacar as limitações do presente estudo, que, por apresentar delineamento transversal, permite uma visão instantânea do desfecho e exposição, fato que não possibilita compreender temporariamente a relação entre as causas (características sociodemográficas ocupacionais, condições de saúde e de trabalho) com o efeito (acidentes de trabalho). Em adição, o estudo foi realizado com uma população de comerciantes informais existente em área específica do Município de Jequié, não levando em consideração aqueles que desenvolvem suas atividades nas próprias casas ou em ruas da cidade. Futuros estudos de caráter prospectivo são necessários para elucidar a direção das associações encontradas.

\section{Conclusão}

Os acidentes de trabalho entre os trabalhadores informais do comércio apresentaram incidência elevada em comparação com os poucos estudos que abordam tais agravos na economia informal brasileira. Aponta-se para sexo, idade, tipo de mercadoria comercializada, percepção de fatores de risco à saúde no processo de trabalho, esforço físico e demanda psicológica como fatores associados aos acidentes de trabalho.

A análise dos resultados do estudo indica a necessidade de investimento em pesquisas sobre os trabalhadores informais do comércio, buscando-se conhecer melhor as causas e características dos acidentes de trabalho para o delineamento de medidas preventivas e de promoção da saúde. Aponta, ainda, para a necessidade de políticas públicas voltadas para a prevenção de acidentes de trabalho no setor informal comércio, especialmente junto aos trabalhadores do sexo masculino e inseridos em atividades de açougues. 


\section{Resumen}

El objetivo del estudio fue estimar la incidencia de accidentes no mortales en el trabajo entre los trabajadores del comercio informal, y analizar los factores asociados a estos sucesos en términos de condiciones laborales en el trabajo, sociodemográficas y aspectos sanitarios. Un estudio transversal con 434 trabajadores de la zona comercial de la ciudad de Jequié, Bahia, Brasil, en 2013. Se utilizó la regresión logística para evaluar los posibles factores asociados con el accidente. Se estimó la incidencia de accidentes en un 32,3\% (últimos 12 meses), y el análisis multivariado reveló una mayor probabilidad de ocurrencia de accidentes en trabajadores varones $(O R=1,61)$, más jóvenes $(O R=4,62)$, los comerciantes carne / aves de corral $(O R=9,55)$ y que tienen un alto esfuerzo físico $(O R=1,71)$. Los resultados indican la necesidad de políticas públicas para prevenir accidentes en el sector del comercio informal.

Accidentes de Trabajo; Salud Laboral; Comercio

\section{Referências}

1. Ruiz MT, Barboza DB, Soler ZASG. Acidentes de trabalho: um estudo sobre esta ocorrência em um hospital geral. Arq Ciênc Saúde 2004; 11:119-24.

2. Ministério da Saúde. Notificação de acidentes do trabalho fatais, graves e com crianças e adolescentes. Brasília: Ministério da Saúde; 2006. (Série A. Normas e Manuais Técnicos).

3. Iwamoto HH, Camargo FC, Tavares LC, Miranzi SSC. Acidentes de trabalho fatais e a qualidade das informações de seus registros em Uberaba, em Minas Gerais e no Brasil, 1997 a 2006. Rev Bras Saúde Ocup 2011; 36:208-15.

4. Dias EC, Oliveira RP, Machado JH, Minayo-Gomez C, Perez MAG, Hoefel MGL, et al. Employment conditions and health inequities: a case study of Brazil. Cad Saúde Pública 2011; 27:2452-60.

\section{Colaboradores}

M. A. Rios contribuiu na concepção, levantamento bibliográfico, coleta e análise de dados, redação e revisão final do artigo. A. A. Nery colaborou na concepção, redação e revisão crítica do artigo. P. A. A. Rios, C. A. Casotti e J. P. Cardoso contribuíram na análise e interpretação dos dados e na redação e revisão crítica do artigo.

\section{Agradecimentos}

À Fundação de Amparo à Pesquisa do Estado da Bahia (FAPESB) e à Universidade Estadual do Sudoeste da Bahia, pelo financiamento (Edital no 068/2012).
5. Krein JD, Proni MW. Economia informal: aspectos conceituais e teóricos. Brasília: Organização Internacional do Trabalho; 2010. (Série Trabalho Decente no Brasil; Documento de Trabalho, 4).

6. Noronha EG. "Informal", ilegal, injusto: percepções do mercado de trabalho no Brasil. Rev Bras Ciênc Soc 2003; 18:111-73.

7. Oficina Regional para América Latina y el Caribe, Organización Internacional del Trabajo. Panorama laboral 2009. Lima: Organización Internacional del Trabajo; 2009.

8. Cisne AMB, Oliveira LS, Santos PJP, Pinto VRS. Trabalhadores do comércio: como não negociar a saúde. São Paulo: Instituto Brasileiro de Saúde e Meio Ambiente; 2011. (Cadernos de Saúde do Trabalhador). 
9. Lourenço EAS, Bertani IF. Saúde do trabalhador no SUS: desafios e perspectivas frente à precarização do trabalho. Rev Bras Saúde Ocup 2007; 32:121-34.

10. Ministério do Trabalho e Emprego. Guia de análise de acidentes de trabalho. Brasília: Ministério do Trabalho e Emprego; 2010.

11. Santana VS, Cunha S. Estudos transversais. In: Almeida Filho N, Barreto ML, organizadores. Epidemiologia \& saúde. Fundamentos, métodos, aplicações. Rio de Janeiro: Editora Guanabara Koogan; 2011. p. 186-93.

12. Soares JFS. Incidência cumulativa anual de acidentes de trabalho não fatais - estimativas nacionais para o Brasil [Tese de Doutorado]. Salvador: Instituto de Saúde Coletiva, Universidade Federal da Bahia; 2012.

13. Araújo TM, Karasek R. Validity and reliability of the job content questionnaire in formal and informal jobs in Brazil. Scand J Work Environ Health 2008; 6:52-9.

14. Karasek R. Job Content Instrument: questionnaire and user's guide. Lowell: University of Massachusetts; 1985 .

15. Souza SF, Carvalho FM, Araújo TM, Porto LP. Fatores psicossociais do trabalho e transtornos mentais comuns em eletricitários. Rev Saúde Pública 2010; 44:710-7.

16. Hagberg M, Christiani D, Courtney TK, Halperin W, Leamon TB, Smith TJ. Conceptual and definitional issues in occupational injury epidemiology. Am J Ind Med 1997; 32:106-15.

17. Conselho Nacional de Saúde. Resolução no 466, de 12 de dezembro de 2012. Aprova as diretrizes e normas regulamentadoras de pesquisas envolvendo seres humanos. http://conselho.saude.gov.br/ resolucoes/2012/Reso466.pdf (acessado em 11/ Mai/2015).

18. Pohl HH, Reckziegel MB, Reuter EM, Galliano LM, Corbellini VA, Stein MJ. Perfil de saúde dos trabalhadores do comércio: um estudo relacionado com a aptidão física. Revista Brasileira de Pesquisa em Saúde 2013; 15:17-24.

19. Campos A, Tavares JC, Lima V. Prevenção e controle de riscos com máquinas, equipamentos e instalações. São Paulo: Editora Senac; 2010.

20. Rios MA, Nery AA, Alves MS, Jesus CS. Acidentes e doenças relacionadas ao trabalho em Jequié, Bahia, registrados no Instituto de Seguridade Social, 2008-2009. Epidemiol Serv Saúde 2012; 21:315-24.

21. Ascari RS, Zatti CA. O perfil dos acidentes de trabalho fatais na Região Sul do Brasil no ano de 2010. Revista Uningá Review 2013; 15:18-22.

22. Bortoleto MSS, Nunes EFPA, Haddad L, Reis GAX. Acidentes de trabalho em um pronto atendimento do Sistema Único de Saúde em município de médio porte da região sul do Brasil. Espaço para a Saúde 2011; 13:91-7.

23. Alves EF. Perfil dos acidentes de trabalho no Brasil, 2004/2007. Revista Saúde e Pesquisa 2010; 3:297-302.
24. Departamento Intersindical de Estatística e Estudos Socioeconômicos. O jovem comerciário: trabalho e estudo. Boletim Trabalho no Comércio 2009; 1:1-5.

25. Fehlberg MF, Santos I, Tomasi E. Prevalência de fatores associados a acidentes de trabalho em zona rural. Rev Saúde Pública 2011; 35:269-75.

26. Motta PT, Carvalho RLR, Duarte MEL, Rocha ADM. Análise dos acidentes de trabalho do setor de atividade econômica comércio no município de Belo Horizonte. REME Rev Min Enferm 2011; 15:427-33.

27. Cardoso JP, Araújo TM, Carvalho FM, Oliveira NF Reis EJFB. Aspectos psicossociais do trabalho e dor musculoesquelética em professores. Cad Saúde Pública 2011; 27:1498-506.

28. Farias MD, Araújo TM. Transtornos mentais comuns entre trabalhadores da zona urbana de Feira de Santana-BA. Rev Bras Saúde Ocup 2011; 36:25-39.

29. Sultan-Taïeb H, Lejeune C, Drummond A, Niedhammer I. Fractions of cardiovascular diseases, mental disorders and musculoskeletal disorders attributable to job strain. Int Arch Occup Environ Health 2011; 84:911-25.

30. Karasek RA, Theörell T. Healthy work-stress, productivity, and the reconstruction of working life. New York: Basic Books; 1992.

31. Tuomi K, Huuttanen P, Nykyri E, Ilmarien J. Promotion of word ability, the quality of work na retirement. Occup Med 2001; 51:318-24.

32. Pohjonen T, Ranta R. Effects of worksite physical exercise intervention on physical fitness, perceived health satatus, and work ability among home care workers: five-year follow-up. Prev Med 2001; 32:465-75.

33. Cordeiro R. Suggestion of an inverse relationship between perception of occupational risks and work-related injuries. Cad Saúde Pública 2002; 18:45-54.

34. Areosa J, Augusto N. Segurança e saúde comportamental: reflexões preliminares. In: Internationa Symposium on Occupational Safety and Hygiene. Guimarães: Universidade do Minho; 2012. p. 33-5.

35. Areosa J. As percepções de riscos dos trabalhadores: qual a sua importância para a prevenção de acidentes de trabalho? In: Veloso Neto HA, Areosa J, Arezes P, organizadores. Impacto social dos acidentes de trabalho. Vila do Conde: Civeri Publishing; 2012. p. 65-97.

36. Lima ML. Percepção de riscos ambientais. In: Soczka L, organizador. Contextos humanos e psicologia ambiental. Lisboa: Fundação Calouste Gulbenkian; 2005. p. 203-45.

37. Veloso Neto HA. Os acidentes de trabalho como fonte de conhecimento e aprendizagem organizacional. In: Veloso Neto HA, Areosa J, Arezes P, organizadores. Impacto social dos acidentes de trabalho. Vila do Conde: Civeri Publishing; 2012. p. 199-225.

Recebido em 30/Jun/2014

Versão final reapresentada em 19/Dez/2014

Aprovado em 09/Jan/2015 